\title{
Rib Fractures, Flail Chest, and Pulmonary Contusion
}

\author{
K. Shad Pharaon ${ }^{1} \cdot$ Silvana Marasco $^{2} \cdot$ John $_{\text {Mayberry }}^{3}$
}

Published online: 8 September 2015

(C) Springer International Publishing AG 2015

\begin{abstract}
Blunt chest trauma accounts for a significant proportion of debilitating and life-threatening injuries. Rib fractures are notoriously painful and can lead to prolonged hospitalization, contribute to the development of pneumonia and respiratory failure, and delay outpatient recuperation significantly. Flail chest, along with chest wall deformity, the most severe of chest wall injuries, is associated with significant acute morbidity and mortality. Pulmonary contusion often accompanies blunt chest wall trauma and when diffuse will result in respiratory failure regardless of other injuries. Pulmonary lacerations, pneumatoceles, and even lobar infarction can occur. In this review, we describe the development of current principles of management of rib fractures, flail chest, and pulmonary contusion. Emerging or unclarified strategies include the importance of acute pain control of rib fractures to alleviate the development of chronic pain, the role of rib fracture operative reduction/internal fixation (ORIF) in severe chest wall trauma, and the use of surfactant and dual lung ventilation for severe pulmonary contusion.
\end{abstract}

Keywords Rib fracture $\cdot$ Flail chest - Thoracic trauma . Pulmonary contusion $\cdot$ Post-trauma respiratory failure

This article is part of the Topical Collection on Blunt Trauma to the Chest

John Mayberry

john.mayberry@sarmc.org

1 PeaceHealth Southwest Medical Center, Vancouver, WA, USA

2 The Alfred Hospital, Melbourne, VIC, Australia

3 Trauma and Acute Care Surgery, Saint Alphonsus Regional Medical Center, 999 N Curtis Rd Suite 407, Boise, ID 83706, USA

\section{Introduction}

Rib fracture injuries extend across a broad spectrum of severity ranging from a single fractured rib that may be sustained in a fall to multiple fractured ribs that result in a flail chest with respiratory failure. Aside from the acute impact of rib fracture injury, long-term pain, disability and deformity also frequently occur $[1,2,3 \cdot, 4 \bullet]$. Despite this, the management for the vast majority of rib fracture injuries remains supportive only, with analgesia, chest physiotherapy, and, when required, respiratory support being mainstays of care [5••]. Acute management, however, may not address the potential long-term morbidity of such injuries. Trauma patients with multiple fractured ribs demonstrate a clinically significant reduction in quality of life out to 24 months with less than half the thoracic trauma group returning to a good level of functioning at that time [6•]. Pulmonary contusion often accompanies blunt thoracic trauma with or without rib fractures. The severity also ranges from mild to severe. The tendency of pulmonary contusions to "blossom" prior to beginning a trajectory of resolution puts the patient at risk for respiratory deterioration over the subsequent 2 to 3 days. In this review, we detail the important physiologic principles which guide effective management of blunt thoracic injury and provide insight into new approaches being developed.

\section{Rib Fractures: The Importance of Acute Pain Management}

There is mounting evidence that a patient's perception of pain in the early post-injury period is associated with chronic pain development [7]. A recent prospective study of rib fracture patients found that pain and disability at 8 weeks post injury could be predicted by the pain intensity within the first few 
days after injury [8]. Interestingly, the number of fractures and the bilaterality of fractures were not predictive. Thus, pain management in the early post-injury setting is likely paramount to obtaining a more favorable recovery. Opioids, oral or intravenous, are traditional first-line therapy for acute rib fracture pain. But because of chronic misuse potential and central desensitization concerns, pain researchers and clinicians are increasingly recommending that opioids be used only in combination with other analgesic modalities such as acetaminophen, nonsteroidal anti-inflammatory medication (NSAID), the anticonvulsants gabapentin and pregabalin,

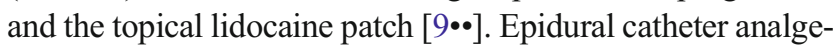
sia can be an effective modality in patients at risk for respiratory deterioration secondary to pain, but injured patients frequently have contraindications preventing insertion $[10,11]$. Continuous intercostal nerve blockade with local anesthetic is available as a bedside procedure and has applicability and benefit for a broader range of patients [12•]. Whether early operative rib fixation is of benefit to reduce acute and chronic pain remains to be determined [13]. Conventional wisdom leads us to believe that acute surgical management has benefit in selected patients with significant fracture displacement [14].

\section{Flail Chest}

Flail chest injury has been associated with a high mortality rates historically and up to $16 \%$ more recently [15-17]. In patients with "physiologic" flail visibly apparent paradoxical chest wall motion leads to inefficient respiratory effort and compression of the lung and diminishes the negative intrathoracic pressure essential for the passive movement of air into the bronchial tree. Atelectasis leads to increased lung resistance and decreased compliance, making the work of breathing much more difficult. Loss of the ability to generate negative intrathoracic pressure with breathing also impairs venous return, a passive process dependent on the negative intrathoracic pressure generated with each breath. In patients without a visible flail segment, i.e., an "anatomic" or "radiologic" flail, the physiologic derangements can be similarly destructive. If the patient survives the early phase of injury, pain, ineffectual cough, and persistent lung atelectasis can lead to pneumonia.

Identification of the high mortality in this condition, and of the physiological derangement, led to early reports of external support by mean of traction years before mechanical ventilation was developed [18]. Strapping, sandbagging, and physical traction by attached weights via pulleys to the patient's skin with forceps were described. Development of the mechanical ventilator and its increasing clinical use in the 1950s led to the concept of internal pneumatic splinting of flail chest injury. Surprisingly, internal pneumatic splinting remained the standard management of flail chest injury for the next 50 years and, in many centers, remains standard management today. Despite scattered reports of surgical open reduction and internal fixation (ORIF) of fractured ribs, lack of dedicated rib prostheses, hardware failures and variable results led to a general lack of acceptance of an operative strategy.

In 2002, however, a small randomized trial was published demonstrating significant benefits of flail chest ORIF in terms of ventilator time, respiratory function, pain, return to work, and treatment costs [19]. Increasing interest in operative fixation of fractured ribs was developing independently in a variety of centers at that time, and over the next few years, a number of specific surgical rib prostheses came onto the market (outlined below). Level 1 evidence demonstrating the benefits of flail chest ORIF has subsequently been generated [20•0]. Non-randomized, cohort-comparison trials have generally confirmed these findings with the caveat that in patients with significant pulmonary contusions, flail chest repair is not advised $[21 \bullet$, $22 \bullet \cdot]$. Furthermore, the long-term morbidity arising from conservative management of flail chest injuries has also generated interest in early surgical management.

No prospective randomized controlled trials have yet been done in other patient groups. Selected patients with multiple displaced fractured ribs without a flail segment. non-ventilatordependent flail chest patients, patients with simple fractured rib injuries such as sporting injuries; and patients with chronic pain or malunion of old rib fractures have been reported in case series to benefit from ORIF [23, 24••]. The selection criteria for rib fixation in these groups need further investigation. Table 1 summarizes current accepted indications for rib fracture ORIF.

Table 1 Current indications for rib fracture ORIF

1. Flail chest with failure to wean from ventilator inclusion criteria:

(a) Paradoxical movement visualized or

(b) Anatomic flail (3 or more consecutive ribs fractured in 2 or more places) and

(c) No significant pulmonary contusion

(d) No significant brain injury

2. Chest wall defect/hernia/severely displaced fractures (Fig. 1) or chest wall implosion syndrome [14] inclusion criteria:

(a) Non-repair of defect may result in pulmonary hernia or

(b) Severely displaced fractures are significantly impeding lung expansion in that hemithorax and

(c) Patient is expected to survive any other injuries

3. "On the way out" or "thoracotomy for other reason" Inclusion criteria

(a) Significant fracture displacement or distraction and

(b) Patient is expected to survive other injuries

4. Symptomatic rib fracture nonunion (Fig. 2) [24••] inclusion criteria:

(a) CT scan evidence of fracture nonunion at least 4 months post-injury

(b) Chronic pain not improving

(c) Significant impairment of activity level 

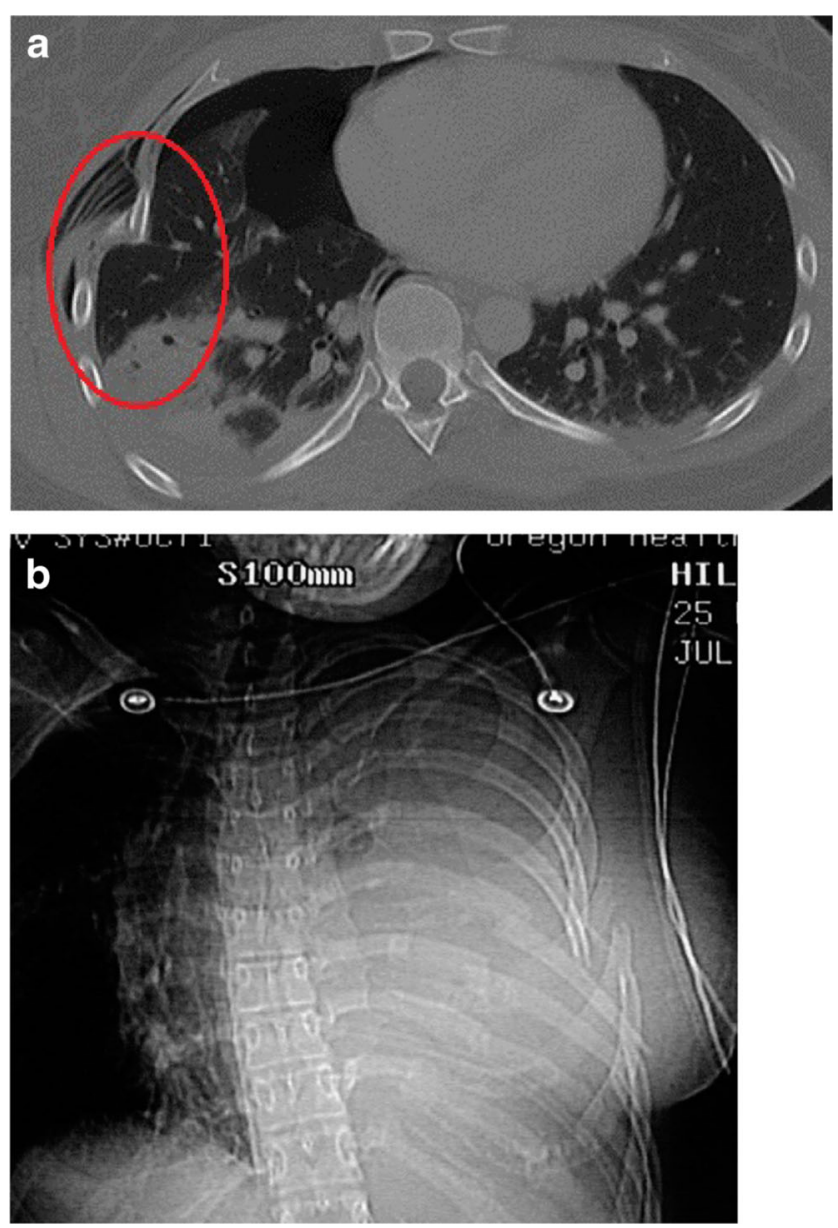

Fig. 1 a CT scan appearance of acute chest wall deformity associated with hemopneumothorax and focal pulmonary contusion following snowmobile crash. b Chest radiograph appearance of left chest wall deformity post assault

\section{Technical Challenges of Rib Fracture ORIF}

Rib fracture ORIF is a technically challenging procedure with which most surgeons caring for injured patients have little

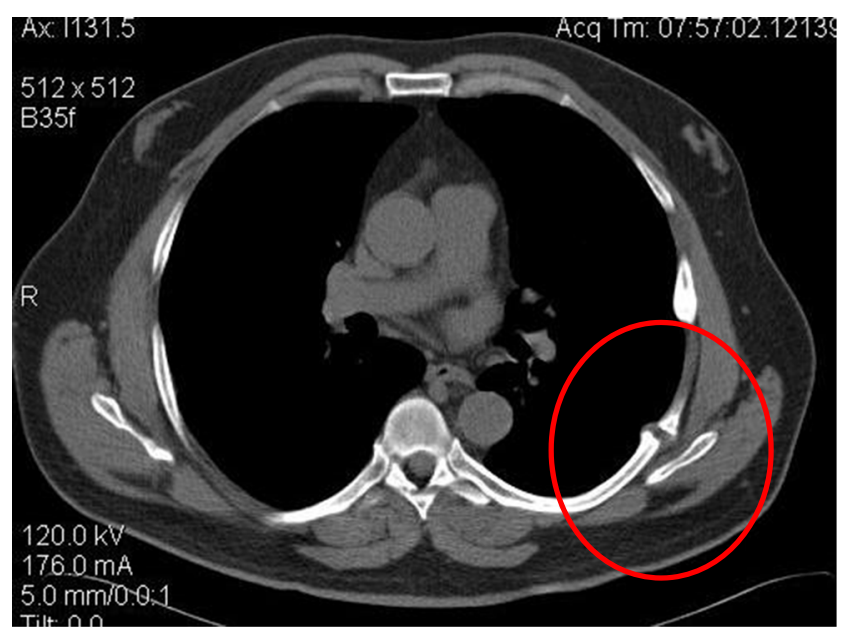

Fig. 2 CT scan appearance of left rib fracture nonunion experience. Because human ribs have a relatively thin (1$2 \mathrm{~mm}$ ) cortex surrounding soft marrow, they are not expected to hold a cortical screw as well as bone with a thicker cortex. In addition, ribs cannot be immobilized during the healing process; thus, any fixation technique must be able to withstand the forces generated by deep breathing and coughing. Surgeons learning this procedure should thus be well-versed in the current concepts of fracture healing and prosthesis fixation.

There are two mechanisms of fracture healing [25]. Primary healing, also referred to as direct, occurs with rigid immobilization and compression at the fracture site. There is essentially no motion at the fracture site, and healing occurs through intramembranous ossification. Rigid immobilization is achieved with conventional plates and compression screws. Secondary healing, also known as indirect, occurs when there is motion at the fracture site. In this setting, if the fracture is adequately healing, a callus should be seen on radiographic imaging. Indirect healing occurs with casting, external fixation, intramedullary nailing, and newer types of locked plating systems. Current rib fracture-specific ORIF plates promote secondary/indirect healing.

The difference between primary and secondary healing illustrates an important concept. Fracture care requires a balance between motion and stability. Inadequate stabilization allows excess motion at the fracture site that may impede tissue differentiation to healthy bone. Over-stabilization is also not desired since when there is no motion at the fracture site, there is no sufficient stress stimulus to promote formation of durable bone.

Open fractures, bone loss, ischemia, contamination, and infection predispose to fixation complications. Negative patient risk factors include advanced age, comorbidities, malnutrition, and smoking [26]. Comorbid conditions such as diabetes mellitus, peripheral vascular disease, rheumatoid arthritis, and vitamin D deficiency are known to impair healing [26, 27]. Perioperative hyperglycemia is an independent risk factor for surgical site infection even in patients without a known history of diabetes [28]. Smoking increases the risk of fracture nonunion [29]. Because of reliable efficacy in inflammation and pain control, NSAID use is prevalent in acute and chronic musculoskeletal injuries, but at a price since NSAIDs have a negative effect on fracture healing [30]. Surgeons offering rib fracture ORIF to injured patients should take these factors into consideration when describing the risk-benefit analysis to the patient during informed consent.

In complex cases involving rib fracture comminution, bone loss, or infection, the use of bone substitutes and grafting has been described [31, 32]. There are many different products and strategies for the encouragement of bone healing in difficult situations. Osteoinductive substances provide growth factors to encourage mesenchymal osteoprogenitor cells to differentiate into the osteoblastic lineage. Osteoconductive grafts provide a scaffold for bone growth. An osteogenesis strategy 
involves the direct transplant of osteoblasts and periosteal cells to the fracture site to produce bone. The gold standard for bone graft had until recently been the autogenous iliac crest bone, which includes all three biologic properties. However, harvesting iliac crest graft results in a relatively high incidence of chronic pain and there is a limited supply. Alternatives to an autograft are cadaveric bone, demineralized bone matrix, and bone morphogenic protein products. Cadaveric bone, however, only has osteoconductive properties. Demineralized bone matrix is commercially available as a putty, paste, sheets, and pieces and provides a degradable matrix of bone proteins, calcium, phosphates, and trace cellular debris that has both osteoconductive and osteoinductive properties [33]. Recombinant bone morphogenic protein (rBMP) is osteoinductive and has been shown to induce bone formation in a variety of clinical scenarios [34]. Surgeons may consider the use of these products in complex rib fracture ORIF situations although minimal experience has been reported.

\section{Rib Fracture ORIF Prostheses}

Several plating systems customized specifically for rib fractures are now available and have been shown safe and effective in clinical trials. This review is not expected to be a complete review of all prostheses available since this is an evergrowing list. Low-profile, anatomically contoured plates with locking screws are a modification of the traditional anterior plating systems used with success throughout the skeletal system [35]. A U-shaped plate designed to overcome the inherent fragility of the human rib by grasping the rib over its superior margin and by securing the plate with anterior to posterior locking screws has also been developed [36]. A novel, screwless system consisting of flexible titanium rib clamps and connecting plates has also been evaluated extensively [37]. Absorbable plates are an attractive alternative to titanium for rib fracture fixation $[20 \bullet \bullet, 38]$. The plates consist of a polylactide polymer with absorption expected by hydrolysis within months. Improved bone healing by the prevention of stress shielding, the allowance for micromotion, and the ability to secure comminuted fractures has been touted [39]. Posterior and more displaced rib fractures are not good candidates for the use of absorbable plates because of the need for more secure fixation [40].

\section{Pulmonary Contusion}

Pulmonary contusion is frequently seen in patients suffering high-velocity civilian trauma $[41 \cdot \bullet]$. In combat or terrorist incident settings, explosions and high-velocity projectiles are additional causes. Pulmonary contusion occurs when there is a transfer of high energy to the lung and can present without obvious external chest wall injury. It is detected either with plain radiography or with computed tomography $(\mathrm{CT})$ although CT is more sensitive. Radiographic evidence of a pulmonary contusion may not become apparent until 24 to $48 \mathrm{~h}$ after the injury. Parenchymal manifestations include capillary hemorrhage, increased capillary permeability, and migration of neutrophils into the extravascular space. This leads to alveolar consolidation, reduced lung compliance, ventilation/ perfusion mismatch with right-to-left shunting, hypercarbia, increased work of breathing, and hypoxia [42]. The composition of surfactant is also impacted. Percentages of phosphatidylcholine and sphingomyelin are significantly higher in the contused lungs than in normal [43]. Adjunctive exogenous bovine surfactant replacement improved oxygenation and decreased ventilator requirements in patients with pulmonary contusions and hypoxemia [44]. Patients with a pulmonary contusion compromising greater than $20 \%$ of their total lung volume are at high risk for pneumonia and acute respiratory distress syndrome (ARDS) [45]. Fortunately, the respiratory derangements associated with pulmonary contusions resolve in most patients in 5 to 7 days and only the rare patient with a high burden of contusions progresses to long-term ARDS.

Treatment of pulmonary contusion is initially supportive by maintaining euvolemia and by promoting pulmonary hygiene with incentive spirometry, chest physiotherapy, and nasotracheal suctioning. Noninvasive ventilation may be attempted prior to endotracheal ventilation in selected patients. Patients who require invasive ventilator support will have additive risks of barotrauma and ventilator-associated pneumonia.

Synchronized intermittent mechanical ventilation (SIMV) with positive end-expiratory pressure (PEEP) is an acceptable mode of ventilation for most patients with pulmonary contusion. SIMV allows patients to breathe spontaneously and avoid hypocapnia, and PEEP increases functional residual capacity by minimizing alveolar collapse during expiration. Rotational bed therapy may promote maintenance of injured lung expansion and prevent the development of pneumonia [46]. If mechanical ventilation is insufficient in improving oxygenation and ventilation, then prone positioning, nitrous oxide supplementation, high-frequency oscillatory ventilation, and extracorporeal membrane oxygenation (ECMO) should be considered. A rare patient with severe unilateral pulmonary contusion may benefit from dual lung ventilation with disparate modes applied to each lung [47]. This will prevent iatrogenic barotrauma in the uninjured lung from the higherpressure settings necessary to inflate the injured lung. If a patient with pulmonary contusion also meets criteria for flail chest ORIF, most agree that this should only be attempted after resolution of pulmonary contusion [48]. Patients with a unilateral pulmonary contusion and flail chest may not tolerate the "good lung down" lateral positioning necessary in the operating room to address the injured hemichest and the 
benefit of early extubation seen in patients with flail chest without pulmonary contusion is less likely to materialize. Patients with flail chest with pulmonary contusion are more likely to require mechanical ventilation, spend more time on the ventilator, have longer ICU and hospital stays, have higher rates of tube thoracostomy placement, and have higher risk of pneumonia [17]. Prophylactic antimicrobials and corticosteroids are not believed to be beneficial and may cause harm. Corticosteroids may contribute to decreased bacterial clearance and subsequent increases in the risk of pneumonia [41].

Additional complications associated with pulmonary contusion are pulmonary lacerations, pneumatoceles, intrabronchial hemorrhage, and lobar infarction. Lacerations will lead to hemopneumothorax requiring tube thoracostomy but generally heal without operative intervention. Pneumatoceles range from small to large and can appear very dramatic on CT scan, but again, they generally do not require intervention. A pneumatocele may enlarge on positive pressure ventilation. Intrabronchial hemorrhage occurs in a minority of patients with pulmonary contusion and is usually self-limiting but in extreme cases may require angiographic embolization or surgical intervention. A rare patient with pulmonary contusion will develop a lobar infarction that requires operative resection.

\section{Conclusion}

Blunt chest trauma often results in serious chest wall and pulmonary injuries including multiple rib fractures, flail chest, and pulmonary contusion. The vast majority of patients with these injuries are managed appropriately with pain control, aggressive respiratory therapy, and mobilization. The prognosis for recovery is good although chronic pain due to chest wall injury is common. A minority of patients with chest wall or pulmonary injuries will require endotracheal intubation and more invasive therapy such as operative flail chest repair or pulmonary resection. The exact role of rib fracture ORIF in polytrauma patients is currently under investigation but accepted indications include chest wall defect/pulmonary herniation, flail chest, and selected instances of severely displaced, multiple rib fractures. A small minority of rib fracture patients will develop a symptomatic nonunion which may be amenable to operative resection or plating with or without bone grafting. Pulmonary contusion is a relative contraindication to rib fracture ORIF depending on the severity of the pulmonary injury. Pneumatoceles are common among patients with significant pulmonary contusion but rarely require operative intervention.

\section{Compliance with Ethics Guidelines}

Conflict of Interest Dr. Marasco declares speaking and travel fees from DePuy Snythes. Dr. Mayberry declares that he is a consultant for Acute
Innovations and receives speaking and travel fees from DePuy Synthes. Dr. Pharaon declares no conflict of interest.

Human and Animal Rights and Informed Consent This article does not contain any studies with human or animal subjects performed by any of the authors.

\section{References}

Papers of particular interest, published recently, have been highlighted as:

- Of importance

•- Of major importance

1. Landercasper J, Cogbill TH, Lindesmith LA. Long-term disability after flail chest injury. J Trauma. 1984;24:410-4.

2. Beal SL, Oreskovich MR. Long-term disability associated with flail chest injury. Am J Surg. 1985;150:324-6.

3. Shelat VG, Eileen S, John L, et al. Chronic pain and its impact on quality of life following a traumatic rib fracture. Eur J Trauma Emerg Surg. 2012;38:451-5. At a minimum of 1 year post injury $\mathbf{2 2 \%}$ of rib fracture patients complained of chronic pain.

4. Gordy S, Fabricant L, Ham B, et al. The contribution of rib fractures to chronic pain and disability. Am J Surg. 2014;207:659-63. At 6 months post injury $22 \%$ and $53 \%$ of rib fracture patients had documented chronic pain and disability.

5.• Todd SR, McNally MM, Holcomb JB, et al. A multidisciplinary clinical pathway decreases rib fracture-associated infectious morbidity and mortality in high-risk trauma patients. Am J Surg. 2006;192:806-11. This landmark study established a multidisciplinary clinical pathway for patients greater than $\mathbf{4 5}$ years of age with 4 or more rib fractures. Pain service consultation, a volume expansion protocol performed by respiratory therapy, physical therapy, and a nutrition consult decreased the historical incidence of mechanical ventilation days, length of stay, infectious morbidity and mortality.

6. Marasco S, Lee G, Summerhayes R, Fitzgerald M, Bailey M. Quality of life after major trauma with multiple rib fractures. Injury. 2015;46:61-5. Quality of life for rib fracture patients was significantly reduced for up to 2 years post injury.

7. Katz J, Jackson BP, Sandler AN. Acute pain after thoracic surgery predicts long-term post-thoracotomy pain. Clin J Pain. 1996;12:50 5.

8. Fabricant L, Ham B, Mullins R, Mayberry J. Prolonged pain and disability are common after rib fractures. Am J Surg. 2013;205: 511-6.

9.• Radresa O, Chauny J, Lavigne G, et al. Current views on acute to chronic pain transition in post-traumatic patients: risk factors and potential for pre-emptive treatments. J Trauma Acute Care Surg. 2014;76:1142-50. This review details pain management modalities effective for the relief of acute post-traumatic pain and the prevention of chronic pain development.

10. Bulger EM, Edwards T, Klotz P, et al. Epidural anlagesia improves outcome after multiple rib fractures. Surgery. 2004;136:426-30.

11. Carrier FM, Turgeon AF, Nicole PC, et al. Effect of epidural analgesia in patients with traumatic rib fractures: a systemic review and meta-analysis of randomized controlled trials. Can J Anaesth. 2009;56:230-42.

12. Truitt MS, Murry J, Amos J, et al. Continuous intercostal nerve blockade for rib fractures: ready for primetime? J Trauma. 2011;71:1548-52. Continuous intercostal nerve blockade 
significantly improved pulmonary function, pain control and shortened length of stay.

13. de Moya M, Bramos T, Agarwal S, et al. Pain as a indication for rib fixation: a bi-institutional pilot study. J Trauma. 2011;71:1750-4.

14. Solberg BD, Moon CN, Nissim AA, et al. Treatment of chest wall implosion injuries without thoracotomy: technique and clinical outcomes. J Trauma. 2009;67:8-13.

15. Cannon RM, Smith JW, Franklin GA, et al. Flail chest injury: are we making any progress? Am Surg. 2012;78:398-402.

16. Pressley CM, Fry WR, Philp AS, et al. Predicting outcome of patients with chest wall injury. Am J Surg. 2012;204:910-4

17. Dehghan N, de Mestral C, McKee MD, et al. Flail chest injuries: a review of outcomes and treatment practices from the National Trauma Data Bank. J Trauma Acute Care Surg. 2014;76:462-8.

18. Bemelman M, Poeze M, Blokhuis TJ, Leenen LPH. Historic overview of treatment techniques for rib fractures and flail chest. Eur J Trauma Emerg Surg. 2010;36:407-15.

19. Tanaka H, Yukioka T, Yamaguti Y, et al. Surgical stabilization of internal pneumatic stabilization? A prospective randomized study of management of severe flail chest patients. J Trauma. 2002;52:727-32.

20.• Marasco SF, Davies AR, Cooper J, et al. Prospective randomized controlled trial of operative rib fixation in traumatic flail chest. J Am Chem Soc. 2013;216:924-32. In this randomized trial of 46 patients with flail chest, operative rib fixation reduced noninvasive ventilation days post extubation, need for tracheostomy, and intensive care unit stay.

21.• Slobogean GP, MacPherson CA, Sun T, et al. Surgical fixation vs nonoperative management of flail chest: a meta-analysis. J Trauma. 2013;216:302-11. A meta-analysis of $\mathbf{1 1}$ studies with a total of 753 patients concluded that flail chest operative fixation may have substantial critical care benefits.

22.• Leinicke JA, Elmore L, Freeman BD, Colditz GA. Operative management of rib fractures in the setting of flail chest: a systematic review and meta-analysis. Ann Surg. 2013;258:914-21. A metaanalysis of 9 studies with a total of 538 patients concluded that flail chest operative fixation was associated with a reduction in days of mechanical ventilation, length of stay, and mortality.

23. de Jong MB, Kokke MC, Hietbrink F, Leenen LPH. Surgical management of rib fractures: strategies and literature review. Scand J Surg. 2014;103:120-5.

24.• Fabricant L, Ham B, Mullins R, Mayberry J. Prospective clinical trial of surgical intervention for painful rib fracture nonunion. Am Surg. 2014;80:580-6. Operative management of chronic rib fracture nonunion was associated with 6 month improvements in RAND 36 Health Survey, McGill Pain Questionnaire, and activity scores. Evidence of intercostal nerve entrapment was present in $38 \%$ of patients.

25. Claes L. Biomechanical principles and mechanobiologic aspects of flexible and locked plating. J Orthop Trauma. 2011;25 Suppl 1:S4-7.

26. Gaston MS, Simpson AH. Inhibition of fracture healing. J Bone Joint Surg (Br). 2007;89:1553-60.

27. Borrelli Jr J, Pape C, Hak D, et al. Physiological challenges of bone repair. J Orthop Trauma. 2012;26:708-11.

28. Richards JE, Kauffmann RM, Obremskey WT, May AK. Stressinduced hyperglycemia as a risk factor for surgical-site infection in nondiabetic orthopedic trauma patients admitted to the intensive care unit. J Orthop Trauma. 2013;27:16-21.

29. Adams CI, Keating JF, Court-Brown CM. Cigarette smoking and open tibial fractures. Injury. 2001;32:61-5.
30. Giannoudis PV, MacDonald DA, Matthews SJ, et al. Nonunion of the femoral diaphysis. The influence of reaming and non-steroidal anti-inflammatory drugs. J Bone Joint Surg (Br). 2000;82:655-8.

31. Nauth A, McKee MD, Einhorn TA, Watson JT, Li R, Schemitsch EH. Managing bone defects. J Orthop Trauma. 2011;25:462-6.

32. Yu NY, Schindeler A, Tagil M, Ruys AJ, Little DG. Use of BMPs and bisphosphonates in improving bone fracture healing. Front Biosci (Elite Ed). 2012;4:2647-53.

33. Gruskin E, Doll BA, Futrell FW, Schmitz JP, Hollinger JO. Demineralized bone matrix in bone repair: history and use. Adv Drug Deliv Rev. 2012;64:1063-77.

34. McKay WF, Peckham SM, Badura JM. A comprehensive clinical review of recombinant human bone morphogenetic protein-2 (INFUSE Bone Graft). Int Orthop. 2007;31:729-34.

35. Bottlang M, Long WB, Phelan D, et al. Surgical stabilization of flail chest injuries with MatrixRIB implants: a prospective observational study. Injury. 2013;44:232-8.

36. Sales JR, Ellis TJ, Gillard J, et al. Biomechanical testing of a novel, minimally invasive rib fracture plating system. J Trauma. 2008;64: $1270-4$

37. Wiese MN, Kawel-Boehm N, de la Santa PM, et al. Functional results after chest wall stabilization with a new screwless fixation device. Eur J Cardiothorac Surg. 2015;47:868-75.

38. Mayberry JC, Terhes JT, Ellis TJ, et al. Absorbable plates for rib fracture repair: preliminary experience. J Trauma. 2003;55:835-9.

39. Viljanen J, Pihlajamaki H, Kinnunen J, Bondestam S, Rokkanen P. Comparison of absorbable poly-L-lactide and metallic intramedullary rods in the fixation of femoral shaft osteotomies: an experimental study in rabbits. J Orthop Sci. 2001;6:160-6.

40. Marasco SF, Sutalo ID, Bui AV. Mode of failure of rib fixation with absorbable plates: a clinical and numerical modeling study. J Trauma. 2010;68:1225-33.

41.• Cohn SM, DuBose JJ. Pulmonary contusion: an update on recent advances in clinical management. World J Surg. 2010;34:1959-70. This is an excellent review article providing more detail on the expected outcomes and sequelae of pulmonary contusion as well recent advances in treatment.

42. Craven KD, Openheimer L, Wood LDH. Effects of contusion and flail chest on pulmonary perfusion and oxygen exchange. J Appl Physiol Respir Environ Exerc Physiol. 1979;47:729-37.

43. Aufmkolk M, Fischer R, Voggenreiter G, et al. Local effect of lung contusion on lung surfactant composition in multiple trauma patients. Crit Care Med. 1999;27:1441-6.

44. Tsangaris I, Galikatsou E, Kostanti E, et al. The effect of exogenous surfactant in patient with lung contusions and acute lung injury. Intensive Care Med. 2007;33:851-5.

45. Miller PR, Croce MA, Bee TK, et al. ARDS after pulmonary contusion: accurate measurement of contusion volume identifies highrisk patients. J Trauma. 2001;51:223-30.

46. Fink MP, Helsmoortel CM, Stein KL, et al. The efficacy of an oscillating bed in the prevention of lower respiratory tract infection in critically ill victims of blunt trauma: a prospective study. Chest. 1990;97:132-7.

47. Cinella G, Dambrosio M, Brienze N, et al. Independent lung ventilation in patients with unilateral pulmonary contusion. Monitoring with compliance and EtCO2. Int Care Med. 2001;27:1860-7.

48. Vana PG, Neubauer DC, Luchette FA. Contemporary management of flail chest. Am Surg. 2014;80:527-35. 